\title{
SEQUELAE OF CHRONIC RHEUMATIC HEART DISEASE AMONG PATIENTS AT TWO TEACHING HOSPITALS IN SINDH, PAKISTAN
}

\author{
Mumtaz Ali Shaikh, Rafi Ahmed Ghori and Bikha Ram Devrajani
}

\begin{abstract}
OBJECTIVE: To evaluate different sequelae in adult patients of chronic rheumatic heart disease. DESIGN: A descriptive study.

SETTING: This study was conducted at Liaquat University Hospital and Isra University Hospital, Hyderabad- Sindh from January 2003 to December 2004.

METHODS: Patients of chronic rheumatic heart disease who presented at Liaquat University Hospital and Isra University Hospital were enrolled in the study. Demographic details of patients such as age, sex, socioeconomic status as well as their symptoms such as dysponea, palpitation and chest pain were noted on a proforma. Number of rheumatic fever attacks in the past were also noted. Patients were examined carefully for cardiac valvular lesions and for signs of acute rheumatic fever. Patients underwent different investigations such as blood complete picture, ESR, ASO titre, ECG, X-ray chest, echocardiography and Doppler. All patients had their calcium levels and collagen profile checked to exclude non rheumatic causes of valvular heart disease.

RESULTS: One hundred adult patients of chronic rheumatic heart disease were studied for various valvular sequelae. Out of 100 patients studied, $49(49 \%)$ patients were males and $51(51 \%)$ were females. Seventy-three (73\%) patients were below 30 years of age (Group A) and $27(27 \%)$ patients were above 30 years of age (Group B). Patients age ranged between 12 and 60 years. Forty-eight (48\%) patients had predominant mitral stenosis. Forty-two (42\%) patients had predominant mitral regurgitation. Nine (9\%) patients had aortic regurgitation and one (1\%) had aortic stenosis along with mitral valve disease.

CONCLUSION: From this study, it is concluded that mitral valve stenosis is frequently observed than mitral regurgitation and aortic valve is least affected, therefore, in every case of chronic rheumatic fever mitral valve should be thoroughly examined.
\end{abstract}

KEYWORDS: Chronic rheumatic heart disease. Sequelae. Mitral stenosis. Mitral regurgitation.

\section{INTRODUCTION}

Rheumatic fever (RF) is an illness predominantly of childhood with major symptoms of arthritis and carditis, a prolonged course and tendency to recur. It is widely held to be an abnormal immune reaction to an infection with a group A beta haemolytic streptococci. Despite decades of intensive study, the nature of this reaction remains obscure and in many ways RF is still an enigma. A better knowledge of the natural history of valvular disease and the advances in surgical techniques are allowing to improve the prognosis of patients with valvular heart disease. At present imaging techniques, particularly Doppler echocardiography, transesophageal echocardiography and 3D transesophageal echocardiography are the main tools to determine the diagnosis and prognosis of patients with valvular heart disease. Consequently, decision making in valvular heart disease is nowadays based on a combination of symptomatic status and echocardiographic findings. ${ }^{1}$ Rheumatic heart disease is leading cause of congestive cardiac failure. ${ }^{2}$ Although, the major cause of acquired heart disease in children; RF has declined dramatically in the Western world since 1920s. In under-developed countries, it remains a major problem with annual incidence rate of 100 to 1,000 per 100,000 childhood age group. ${ }^{3}$ Accurate figures for incidence of chronic rheumatic heart disease are not readily available, but in the West, it occurs in less than 5 per 100,000 population. In the third world, figures up to 6 per 1,000 are observed in school children. ${ }^{4}$ There is lack of recent local studies on this very important and common disease of developing countries, and in our country still this is a major problem for 
valvular heart disease. So this study was designed to guide local clinicians for the sequelae and pitfalls in management of chronic rheumatic fever.

\section{PATIENTS AND METHODS}

This study was conducted on one hundred patients of chronic rheumatic heart disease who presented at Liaquat University Hospital and Isra University Hospital, Hyderabad - Sindh. Data were collected through a pre-designed proforma. Patient's age, sex, symptoms such as dysponea, palpitation and chest pain were recorded. Number of RF attacks in the past was also noted. Details about family members in house and socioeconomic condition of patient were also inquired. Patients were examined carefully for cardiac valvular lesions and for signs of acute RF. Patients underwent different investigations such as blood complete picture, ESR, ASO titre, ECG, x-ray chest, echocardiography and Doppler. All patients had their calcium levels and collagen profile checked to exclude nonrheumatic causes of valvular heart disease. Major diagnostic criteria for RF were carditis, polyarthritis, chorea, erythema marginatum and subcutaneous nodules. Minor criteria for RF included previous episode of RF or rheumatic heart disease, arthralgia, fever, raised ESR, positive test for $\mathrm{C}$-reactive protein or leukocytosis, prolonged PR interval, evidence of recent group A streptococcal infection, raised ASO titre, positive throat swab and recent scarlet fever.

Two major or one major and two minor criteria make RF very likely if supported by evidence of a preceding streptococcal infection. ${ }^{5}$ Cardiac valvular diseases were assessed by echocardiography. M mode and 2D echocardiography and Doppler techniques were used. Views taken were parasternal long axis and short axis, apical four chamber view and subcoastal four chamber views. All four valves were studied in detail and mitral valve area was measured in all the cases. Severity of mitral stenosis was assessed by mitral valve area; mild mitral stenosis as $\left(1.5-2.0 \mathrm{~cm}^{2}\right)$, moderate $\left(1.0-1.5 \mathrm{~cm}^{2}\right)$ and severe $\left(<1.0 \mathrm{~cm}^{2}\right)$. In general, surgery can be differed in patients with mild mitral stenosis i.e. mitral valve area of more than $1-\mathrm{cm}^{2} / \mathrm{m}^{2}$ BSA, where as it should be recommended for those with moderate or severe mitral stenosis i.e. mitral valve area less than $1-\mathrm{cm}^{2} / \mathrm{m}^{2}$ BSA. ${ }^{6}$ Patients of acute rheumatic fever were excluded from the study on the basis of criteria for acute rheumatic fever and investigations. All types of severe valvular lesions after excluding contraindications were advised surgical intervention.
Patients were advised regular follow up visits at out door department.

\section{RESULTS}

One hundred patients of chronic rheumatic heart disease were studied, for assessing the frequency of different valvular lesions. Among these, 49 (49\%) patients were males and 51 (51\%) females. Their ages ranged between 12 and 60 years. Two groups were arranged according to age; Group A with less than 30 years of age and Group B with more than 30 years of age (Table I). Ninety-three (93\%) patients belonged to poor socioeconomic class alongwith 8-10 family members. They were also living in rural areas, in congested homes. Seven patients belonged to middle class family, and lived in urban areas. Past history of RF was present in 40 patients. No any patient was on prophylactic long acting penicillin. Mitral stenosis was diagnosed in 48 patients. Thirty-seven patients were having pure mitral stenosis and 11 had mixed valvular heart disease, predominating mitral stenosis. Among these, $34(70.8 \%)$ patients of mitral stenosis were females and 14 (29.2\%) patients were males. In patients of mitral stenosis, three groups were made according to severity. Severity of mitral stenosis was assessed by mitral valve area, calculated by $2 \mathrm{D}$ echocardiography and Doppler. In Group I, mild mitral stenosis was in $3(6.2 \%)$ patients. Group II included moderate mitral stenosis in 11 (23\%) patients. In group III, severe mitral stenosis was present in $34(70.8 \%)$ patients. Patients with severe mitral stenosis were recommended mitral valve surgery after careful evaluation. Mitral regurgitation was found in 42 patients. Thirty-five patients had pure mitral regurgitation and 7 had mixed valvular heart disease with predominant mitral regurgitation. Among 42 patients, 30 (71.4\%) were males and $12(28.6 \%)$ females. Patients with severe mitral regurgitation and LVEDD reaching 55-mm were recommended mitral valve surgery after careful evaluation. Most of the patients with mitral regurgitation were of severe nature. Chronic rheumatic aortic regurgitation was found in 9 patients, associated with mitral valve disease. Among these 9 patients, 4 were males and 5 females. One patient had aortic stenosis of rheumatic origin (Table II). Five patients had pericardial effusion along with mitral valve disease. Five patients had already undergone mitral valvotomy and same number had undergone mitral valve replacement. 
TABLE I:

AGE AND SEX DISTRIBUTION OF PATIENTS WITH CHRONIC RHEUMATIC HEART DISEASE $(n=100)$

\begin{tabular}{|c|c|c|}
\hline \multicolumn{1}{|c|}{ Age } & \multicolumn{2}{|c|}{ Number of patient } \\
\hline Group A (<30 years) & \multicolumn{2}{|c|}{$73(73 \%)$} \\
\hline Group B (>30 years) & \multicolumn{2}{|c|}{$27(27 \%)$} \\
\hline Sex & Male & Female \\
\hline Group A & $36(36 \%)$ & $37(37 \%)$ \\
\hline Group B & $13(13 \%)$ & $14(14 \%)$ \\
\hline
\end{tabular}

TABLE II:

PATTERN OF SEQUELAE IN PATIENTS WITH CHRONIC RHEUMATIC DISEASE $(n=100)$

\begin{tabular}{|l|c|c|c|}
\hline & Male & Female & Total \\
\hline Mitral stenosis & 14 & 34 & 48 \\
\hline Mitral regurgitation & 30 & 12 & 42 \\
\hline Aortic regurgitation & 4 & 5 & 9 \\
\hline Aortic stenosis & 1 & 0 & 1 \\
\hline
\end{tabular}

\section{DISCUSSION}

Considering the disabling nature of chronic rheumatic heart disease, this study was planned to assess the sequelae in 100 patients of chronic rheumatic heart disease who presented at our departments of medicine. It is well known that RF licks the joints but bites the heart leading to pericarditis, myocarditis and endocarditis. The brunt of chronic heart disease is mostly beard by mitral valve apparatus. Mitral stenosis can result from various causes but the predominant reason for mitral stenosis is chronic rheumatic heart disease. ${ }^{7}$ Mitral stenosis is common in females. In this study, $70.8 \%$ patients of mitral stenosis were females. Transesophageal echocardiography (TEE) has become the standard of care for evaluating valvular procedures, thus providing an immediate gauge of the surgical results and helping to avoid suboptimal surgical outcomes. ${ }^{8}$ The 3D transesophageal echocardiography (TEE) has additional value for patients with mitral valve stenosis undergoing percutaneous mitral balloon valvotomy (PTMV). The 3D TEE method enabled a better description for the mitral valvular anatomy, especially post-PTMV. 3D TEE had additional value over 2-dimensional echocardiography in this group of patients, for selection of patients pre-PTMV, and for analysing pathology of the mitral valve after- ward. ${ }^{9}$ Mitral regurgitation is common in male patients. ${ }^{10}$ It has been reported that mitral valve regurgitation and aortic regurgitation are associated with lower body mass index, an index of body adiposity. ${ }^{11}$ The diagnosis of mitral regurgitation is often made from physical diagnosis, but quantification of severity of valvular dysfunction and timing of intervention are often quite challenging. Refinement of quantitative methods and development of newer technologies, including three-dimensional echocardiography, provide more accurate assessment of mitral anatomy and function. These methods for evaluation of the mitral valve to ascertain etiology or mechanism have important implications for medical or surgical management. ${ }^{12}$ In this study, $71.4 \%$ patients of mitral regurgitation were males. It has been noted that mitral valve is involved in $85 \%$ patients of chronic rheumatic heart disease and in $35 \%$ patients, mitral regurgitation is predominant. ${ }^{13}$ It has been studied that valve repair is highly feasible in selected patients with rheumatic mitral regurgitation, and clinical course is not significantly different from that of patients with degenerative mitral regurgitation. ${ }^{14}$ There is modest agreement in mitral regurgitation and aortic regurgitation assessment between transthoracic echocardiography and prepump tansesophageal echocardiography. Cardiologists, cardiac surgeons, and anesthesiologists must be aware of differences between these methods when using prepump TEE to guide intraoperative decisions. ${ }^{15}$ In this study, $90 \%$ patients had mitral valve disease and $42 \%$ patients had predominant mitral regurgitation. It has been studied that half of patients of mitral stenosis do not give past history of acute rheumatic fever. ${ }^{16}$ In this study, past history of RF was present only in $40 \%$ of patients. In a study at the end of 20 -years, $30 \%$ patients died. Those who were alive, $45 \%$ had evidence of rheumatic heart disease. In all those $45 \%$ patients mitral valve was involved in all the patients except 27 cases. $^{17}$ It is observed that $25 \%$ patients of chronic rheumatic heart disease have pure mitral stenosis and additional $40 \%$ have combined mitral valve disease. ${ }^{18}$ Two-third of all rheumatic mitral stenosis patients were females while $10 \%$ of mitral stenosis patients have additional severe rheumatic aortic regurgitation. ${ }^{19}$ Valvular aortic stenosis is more common in males and very rarely occurs on rheumatic basis. ${ }^{20}$ In this study, only one patient had aortic stenosis along with mitral valve disease. Tricuspid stenosis is present at autopsy in $15 \%$ patients with 
rheumatic heart disease, but is of clinical significance in only about $5 \%$ cases ${ }^{21}$ Rheumatic inflammation of the pulmonic valve is usually associated with involvement of other valves and rarely leads to serious deformity. ${ }^{22}$ One study at Dhaka, Bangladesh has showed that out of 241 cases of valvular heart disease, mitral valve was involved in $61 \%$ cases. Mitral stenosis was the commonest (33.8\%). ${ }^{23}$ Robotically assisted cardiothoracic procedures may allow patients to undergo increasingly complex procedures without the pain and morbidity associated with standard open surgical techniques. The benefits of such an approach are obvious especially since advancing age and multiple co-morbidities are prevalent in patients. ${ }^{24}$ This study had limitations of a limited number of patient sample as well as convenient sampling approach so its findings may be interpreted with caution.

\section{CONCLUSION}

From this study, it is concluded that mitral valve stenosis is observed more frequently than mitral regurgitation and aortic valve is least affected, therefore, in every case of chronic rheumatic fever mitral valve should be thoroughly examined. Hence, it is suggested to establish rheumatic fever units in various hospitals for prevention of this lethal disease and appropriate health education of the patients.

\section{REFERENCES}

1. Gill EA, Pittenger $B$, Otto CM. Evaluation of the severity of valvular heart disease and timing of surgery. Rev Esp Cardiol. 2003;56:900-14.

2. Hafizullah M, Sahibzada WA. Haemodynamic effects of captopril in congestive cardiac failure due to rheumatic heart disease. JPMI. 1995;9:126-9.

3. Akhtar N, Sadiq M, Chagani H, Hafeez A, Rizvi $\mathrm{FH}$, Mehboob M. Guidelines for prevention of rheumatic fever and rheumatic heart disease. Pak J Cardiol. 2004;15(3):136-48.

4. Khalilullah, Ahmed SA, Badsha S, Khan A, Kiani MR. Rheumatic heart disease - a study of surgically excised cardiac valves and biopsies. J Coll Physicians Surg Pak. 2002;12:542-5.

5. Shaikh MA. Cardiac valvular lesions in patients with rheumatic heart disease. J Pak Inst Med Sci. 2004; 15:862-5.

6. Constante Sotelo JL, Mendez Dominguez A. Rheumatic heart disease: cause of cerebrovascu- lar disease at the National Institute of Cardiology "Ignacio Chavez". Arch Cardiol Mex. 2006;76:4751.

7. Roberts WC. Morphologic features of the normal and abnormal mitral valve. Am J Cardiol. 1983; 51:1005.

8. Koch CG, Milas BL, Sayino BS. What does transesophageal echocardiography add to valvular heart surgery? Anesthesiol Clin North Am. 2003;21:587-611.

9. Langerveld J, Valock G, Plokker HW, Ernst SM, Mannaerts HF, Kelder JC, et al. Additional value of three-dimensional transesophageal echocardiography for patients with mitral valve stenosis undergoing balloon valvuloplasty. J Am Soc Echocardiogr. 2003;16:841-9.

10. Periwal KL, Gupta BK, Panwar RB, Khatri PC, Raja S, Gupta R. Prevalence of rheumatic heart disease in school children in Bikaner: an echocardiographic study. J Assoc Physicians India. 2006;54:279-82.

11. Palmieri V, Bella JN, Arnett DK, Oberman A, Kitzman DW, Hopkins PN, et al. Associations of aortic and mitral regurgitation with body composition and myocardial energy expenditure in adults with hypertension: the Hypertension Genetic Epidemiology Network Study. Am Heart J. 2003;145:1071-7.

12. Aikat S, Lewis JF. Role of echocardiography in the diagnosis and prognosis of patients with mitral regurgitation. Curr Opin Cardiol. 2003;18:334-9.

13. Manfrini $O$, Bugiardini R. Rheumatic fever and rheumatic heart disease. G Ital Cardiol (Rome). 2006;7:266-72.

14. Song JK, Kim HS, Song JM, Kang DH, Song H, Choo SJ, et al. Comparison of clinical and echocardiographic outcomes after valve repair: degenerative versus rheumatic mitral regurgitation. J Korean Med Sci. 2003;18:344-8.

15. Neuman YM, Brasch AV, Kobal S, Khan SS, Mirocha JM, Naqvi TZ, et al. Comparison of transthoracic and intraoperative transesophageal color flow doppler assessment of mitral and aortic regurgitation. Cardiology. 2003;145-52.

16. Bland EF, Jones TD. Rheumatic fever and rheumatic heart disease. Circulation. 1951;836:4-9.

17. Kumar A, Sinha M, Sinha DNP. Chronic rheumatic 
heart disease in Ranchi. Angiology. 1982;33:141.

18. Wood $\mathrm{P}$. An appreciation of mitral stenosis. $\mathrm{Br}$ Med J. 1954; 1:1051.

19. Segal J, Harvey WP, Hufhagel CA. Clinical study of one hundred cases of severe aortic insufficiency. A J Med. 1956;21:200.

20. Panidis IP, Segal BL. Aortic valve disease in elderly cardiovascular clinics. Valvular Heart Dis. 1986;5:289-312.

21. Kitchen A, Turner R. Diagnosis and treatment of tricuspid stenosis. Br Heart J. 1964;26:354.

22. Vela JE, Conteras R, Sosa FR. Rheumatic pulmonary valve disease. Am J Cardiol. 1969;23:12.

23. Majumder AA. Echocardiographic pattern of rheumatic valvular disease. An experience at Dhaka Medical College. Pak Heart J. 2002;29:3-4.

24. Shwarts DS, Stames VA. The future is robotically assisted heart surgery. Heart Surgery Dorum. 2003;6:120.

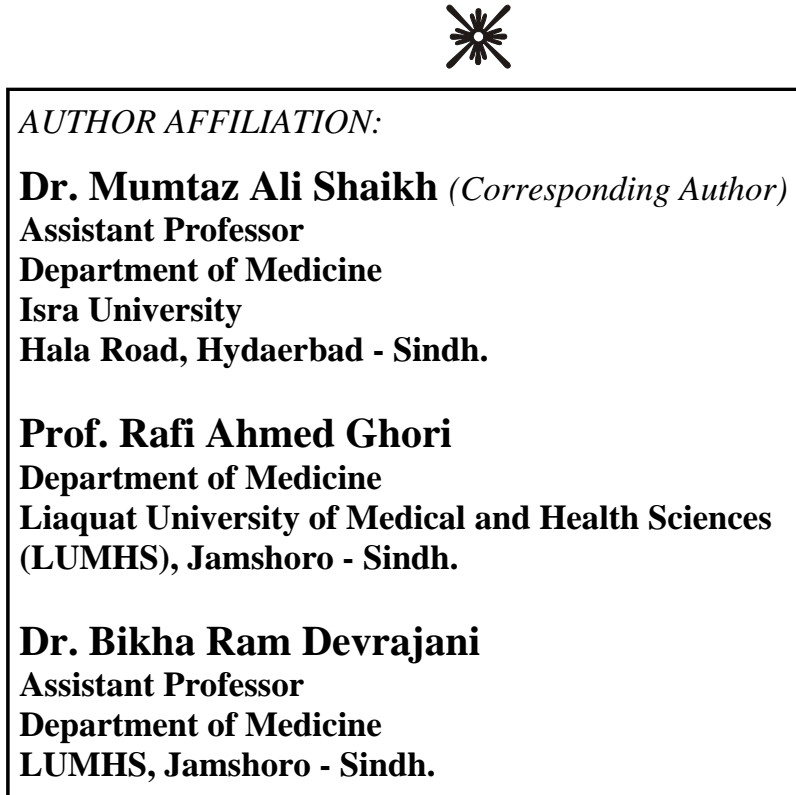

